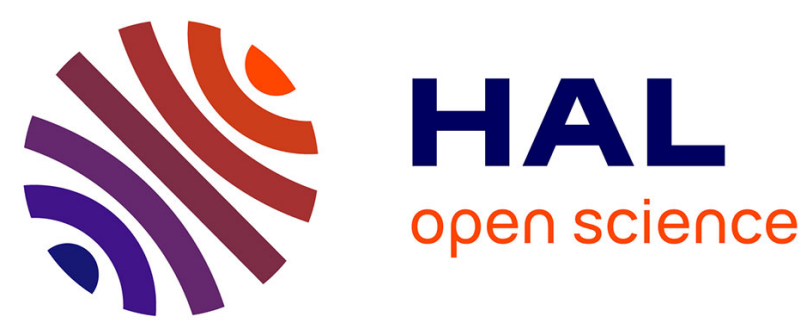

\title{
Fast tailward stream observed in the distant tail associated with substorm: A multi-instrument study
}

S. Rouquette, C. Jacquey, O. Le Contel, A. Lui, D. Williams, R. Mcentire, T. Mukai, V. Angelopoulos, F. Mozer, T. Nagai, et al.

\section{- To cite this version:}

S. Rouquette, C. Jacquey, O. Le Contel, A. Lui, D. Williams, et al.. Fast tailward stream observed in the distant tail associated with substorm: A multi-instrument study. Geophysical Research Letters, 2000, 27 (21), pp.3571-3574. 10.1029/2000GL003811 . hal-02550833

\section{HAL Id: hal-02550833 \\ https://hal.science/hal-02550833}

Submitted on 12 Sep 2020

HAL is a multi-disciplinary open access archive for the deposit and dissemination of scientific research documents, whether they are published or not. The documents may come from teaching and research institutions in France or abroad, or from public or private research centers.
L'archive ouverte pluridisciplinaire HAL, est destinée au dépôt et à la diffusion de documents scientifiques de niveau recherche, publiés ou non, émanant des établissements d'enseignement et de recherche français ou étrangers, des laboratoires publics ou privés. 


\title{
Fast tailward stream observed in the distant tail associated with substorm: a multi-instrument study
}

\author{
Rouquette, S. ${ }^{1}$, C. Jacquey ${ }^{1}$, O. Le Contel ${ }^{2}$, A. T. Y. Lui ${ }^{3}$, D.J. Williams ${ }^{3}$, R.W. McEntire, \\ T. Mukai ${ }^{4}$, V. Angelopoulos ${ }^{5}$, F.S. Mozer ${ }^{5}$, T. Nagai ${ }^{6}$, S.P. Christon ${ }^{7}$, K. Tsuruda ${ }^{8}$, G.D. Reeves ${ }^{9}$
}

\begin{abstract}
We report a fast tailward flow event on July 19 , 1994, observed in the deep tail by the GEOTAIL spacecraft then located at $133 R_{E}$ from the Earth. This event was associated with an isolated substorm and GEOTAII continuously remained inside the plasma sheet during the 2.5 hours period following the substorm onset. Thus, this event provides the opportunity to analyze the substorm dynamics in deep-tail plasma sheet. Using magnetic field (MGF), electric field (EFD), plasma (LEP) and energetic particle (EPIC) data, we show that the fast tailward flow associated with the substorm expansion exhibited clear features: (i) the flow was quasi-stationary during its 2.5 hours long duration; (ii) its structure across the plasma sheet thickness showed two layers associated with two clearly distinct flow regimes. In the central plasma sheet, the plasma speed was roughly constant and uniform. Its value was close to the lobe Alfven velocity. The separation between the two layers precisely took place where the plasma parameter beta was close to one.
\end{abstract}

\section{Introduction}

Both ISEE-3 and GEOTAIL missions have shown that fast tailward flows are often observed in the deep tail in association with substorm expansion (e.g. Hones et al., 1984, Mukai et al., 1998). These flows are generally associated with plasmoids (e.g. Moldwin and Hughes, 1993, Ieda et al., 1998) and with enhanced energetic particle fluxes (e.g. Richardson et al., 1987, Jacquey et al., 1994). In this paper, we report multi-instrument observations obtained onboard GEOTAIL during such a fast tailward flow event, which occurred on July 19,1994. We chose this event because it was associated with an isolated substorm and because GEOTAIL, located in the deep tail at $133 \mathrm{R}_{\mathrm{E}}$ from the Earth, remained inside the plasma sheet during the 2.5 hours duration of the flow. Using magnetic field, electric field, plasma and energetic particle data, we show that the flow was dominated by the particles, could be described by using the hydromagnetic approximation and exhibited a clear spatial structure.

${ }^{1}$ CESR,Toulouse, France.

${ }^{2}$ CETP, Vélizy, France.

${ }^{3}$ APL, JHU, MD, USA.

${ }^{4}$ ISAS, Japan.

${ }^{5}$ SSL, UCB, USA.

${ }^{6}$ Tokyo Inst. Tech., Japan.

${ }^{7}$ UMD, MD, USA.

${ }^{8}$ STEL, Japan.

${ }^{9}$ LANL, NM, USA.

Copyright 2000 by the American Geophysical Union.

Paper number 2000GL003811.

0094-8276/00/2000GL003811 $\$ 05.00$

\section{Large scale observations}

Figure 1 shows five hours of data obtained on the ground and onboard GEOTAIl on July 19, 1994. The AL index displayed in the top panel is an estimator of the substorm westward electrojet intensity. The $400 \mathrm{nT}$ negative bay starting at 05:48:20 UT \pm 30 s, time marked by the vertical dashed line, consists of the usual signature of an isolated substorm. A few minutes after the ground onset time, an energetic electron injection was detected at geosynchronous orbit by both 1989_046 and 1990_095 LANL satellites then located at 18.6 and $3.3 \mathrm{MLT}$ (not shown). Detailed

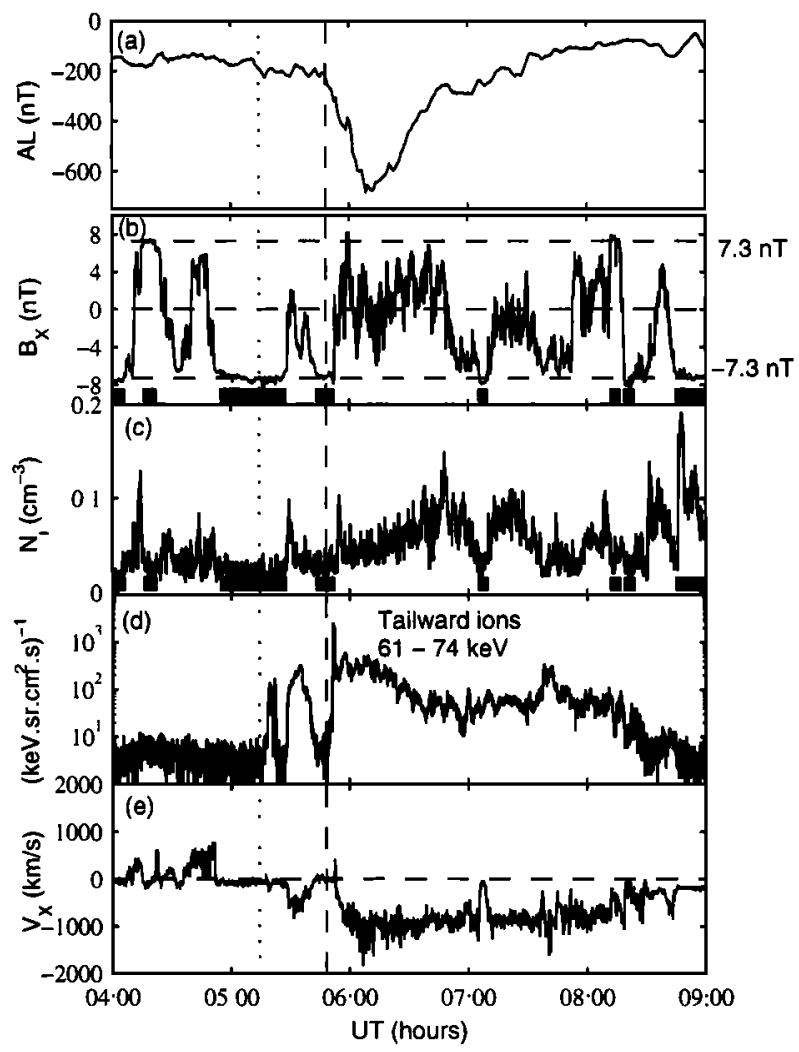

Figure 1. Five hours of data obtained on the ground and at GEOTAIl orbit $\left(\mathrm{X}_{\mathrm{GSE}}=-133 \mathrm{R}_{\mathrm{E}}\right)$. Panel $(\mathrm{a}): 1$ minute $\mathrm{AL}$ index variations. The onset of the substorm is represented by the vertical dashed line; (b): $\mathrm{B}_{\mathrm{X}}$ component of the magnetic field; (c): Ion umber density. The shadowed area is drawn for density values between 0.02 and $0.04 \mathrm{~cm}^{-3}$; (d): Differential flux of 61-74 keV tailward ions; (e): $V_{x}$ component of the plasma bulk velocity. The black areas at the bottom of panels (b) and (c) represent periods when GEOTAII was located in the lobes, defined by $B_{X} \sim 7.3 \mathrm{nT}$. The dashed (dotted) vertical line indicates the substorm onset time (moderate electrojet activation). 
examination of the magnetograms recorded in the whole night sector indicates that a moderate electrojet intensification was observed at 05:14:20 UT (dotted vertical line) prior to the substorm onset.

At 06:00 UT, GEOTAIl was located at $\mathrm{X}_{\mathrm{GSM}} \sim-133 \mathbf{R}_{\mathrm{E}}$, $Y_{G S M} \sim 6.5 R_{E}$ and $Z_{G S M} \sim-4.4 R_{E}$. The $B_{X}$ component of the magnetic field recorded by the MGF instrument (Kokubun et al., 1994) is given in panel (b). Due to the magnetic field gradient inside the plasma sheet, $\mathrm{B}_{\mathrm{X}}$ is indicative of the spacecraft location along the $Z$-direction relative to the plasma sheet. During the periods marked by the horizontal bars the absolute value of $B_{X}$ sustained a weakly varying maximal level of about $7.3 \mathrm{nT}$. These periods are indicative of the satellite being in the lobes, as confirmed by the particle and spacecraft potential data (not shown). Conversely, when the absolute value of $B_{X}$ took smaller values, the satellite was inside the plasma sheet. At 05:52 UT GEOTAIL penetrated inside the plasma sheet and then remained almost continuously inside the plasma sheet during about two hours and a half, sampling the entire plasma sheet thickness.

The ion density measured by the LEP instrument (Mukai et al., 1994) is shown in panel (c). From 04:00 to 08:30 UT, for all time periods when the spacecraft was located inside the lobes, the ion density was between 0.02 and $0.04 \mathrm{~cm}^{-3}$. Moreover, the magnetic field magnitude was very stable during the entire day for all the excursions of the satellite into the lobes. This suggests that the lobe conditions did not change much during the presented period.
Panel (d) displays tailward 61-74 keV energetic ion fluxes measured by the EPIC-ICS instrument (Williams et al., 1994). Detailed analysis of the species and charge state with the help of EPIC data showed that the detected ions consisted of particles of both solar wind and ionospheric origins. Between 04:00 and 05:00 UT, GEOTAIL crossed the plasma sheet four times but the EPIC instruments only detected very low energetic ion fluxes, close to the background level. Between 05:15 and 05:30 UT, two successive tailward energetic ion flux enhancements were observed, associated with a tailward plasma flow. They are believed to be associated with the moderate electrojet intensification observed from the ground prior to the substorm onset. At 05:51:09 UT, i.e. less than 3 minutes after the ground substorm onset, a strong tailward energetic ion burst was observed. Thereafter, the energetic ion flux remained continuously enhanced during more than two hours and a half. Less than 4 minutes after the ground onset time, the tailward plasma bulk velocity increased until a maximum value up to $1500 \mathrm{~km} / \mathrm{s}$ at $06: 07 \mathrm{UT}$. Then, the plasma velocity decreased and reached a value of $900 \mathrm{~km} / \mathrm{s}$ at 06:15 UT. From 06:15 to 08:30 UT the velocity remained roughly constant. We defined this period as the post-onset flow period described in the next section.

\section{Features of the post-onset flow}

Figure 2 shows three hours of data obtained onboard GEOTAIL. Panel (a) displays the angular distribution of the 61-

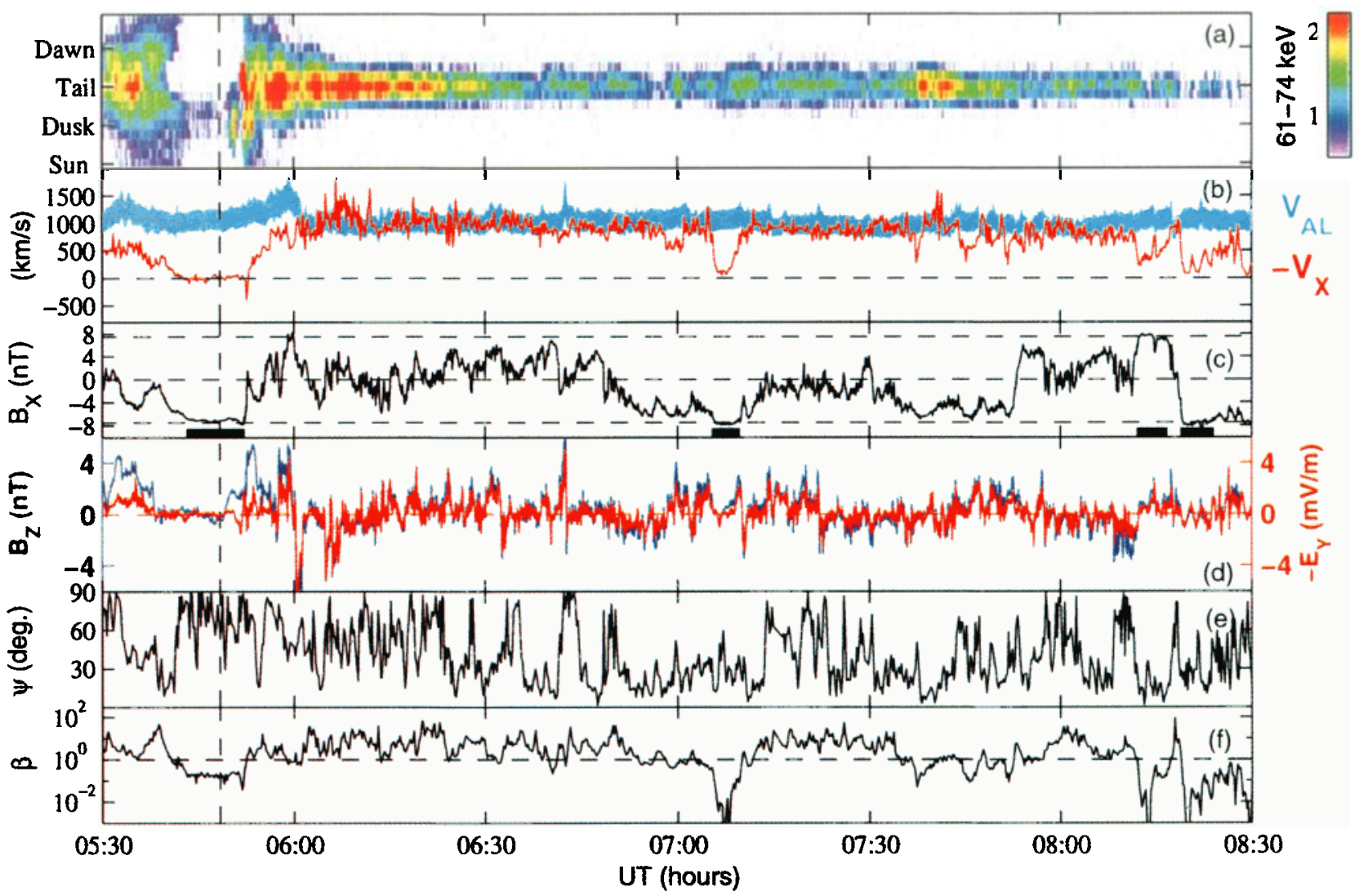

Figure 2. Three hours of data obtained onboard GEOTAIL. Panel (a): Angular distribution of 61-74 keV ions in the equatorial [X-Y]GSE plane. The color scale gives the log of differential flux. The labels of the Y-axis designate the direction of the particles; (b): tailward component of the plasma bulk velocity (red line) and lobe Alfven velocity range (blue shadowed area); (c): $B_{X}$ component of the magnetic field; (d): $B_{Z}$ component of the magnetic field (left axis) and $\mathrm{E}_{\mathrm{Y}}$ component of the electric field (right axis); (e): Variation of the angle $\psi$ (see text); Panel (f): $\beta$ plasma parameter. 
$74 \mathrm{keV}$ ion differential flux measured by EPIC-ICS in the equatorial $[\mathrm{X}-\mathrm{Y}] \mathrm{GSE}$ plane. The labels of the $\mathrm{Y}$-axis designate the direction of the particles. The opposite of the $V_{X}$ component of the plasma velocity measured by LEP is shown in panel (b). The shadowed band represents the lobe Alfven velocity. Its estimation is described in section 4 . The $B_{X}$ component of the magnetic field displayed in panel (c) provides information on the location of the spacecraft in the plasma sheet. At the substorm onset time marked by the vertical dashed line, GEOTAIL was in the lobe. The EPIC instrument detected first at 05:49:30 UT duskward oriented energetic ions indicative of gradient effect (e.g. Williams, 1981). Then at 05:51:09 UT, i.e. 2.5 minutes after the ground onset, enhanced energetic ion fluxes were detected. They first consisted of a strong tailward directed energetic ion burst superimposed on the pre-existing energetic population of the plasma sheet. At the burst detection time, the plasma velocity along the X-direction was very low as indicated by the LEP measurements. Thereafter, the ion anisotropy became more and more tailward oriented until it was highly collimated in the tailward direction at about 06:15 UT. During the two hours following that time, the particle measurements exhibited quasistationary features: (i) the plasma bulk flow was continuously tailward directed and sustained roughly constant value of 900 $\mathrm{km} / \mathrm{s}$ close to the lobe Alfven speed and (ii) the energetic ion flux anisotropy also did not vary much and remained strongly tailward directed. The flow direction of particles appears to have not been affected by the local magnetic field, which was highly variable. Both plasma and energetic ions flew parallel or perpendicular to the magnetic field as well, as shown by the $\Psi$ angle displayed in panel (e). Here, $\Psi$ is the angle between the flow direction relative to the local magnetic field, $\Psi=\operatorname{acos}(\mid \mathbf{V} . \mathbf{B} / \mathrm{VB})$.

The opposite of $E_{Y}$ component measured by the EFD instrument (Tsuruda et al., 1994) has been superimposed on the $B_{Z}$ in panel (d). A strong anti-correlation between $E_{Y}$ and $B_{Z}$ was observed during the whole post-onset flow period. This anticorrelation was observable up to the highest time resolution of the data ( 3 seconds). The ratio $E_{Y} / B_{Z}$ gives a velocity of about $700 \mathrm{~km} / \mathrm{s}$, i.e. close to the plasma bulk flow $(900 \mathrm{~km} / \mathrm{s})$ measured by the LEP instrument inside the central plasma sheet. The discrepancy between these two values is consistent with the one expected to result from the spacecraft potential (Pedersen et al., 1984). Thus, this suggests that the electric field may be regarded as a purely motional field and that the jet can be described by using the hydromagnetic approximation $(\mathbf{E}=-\mathbf{V x B})$.

As shown in panel (e), the plasma $\beta$ parameter (ratio of the thermal ion pressure over the magnetic pressure) was almost continuously greater than 1 (sometimes reaching large values close to 100) during the fast flow event except for some short excursions of the spacecraft into the lobes. The total plasma $\beta$ parameter (ratio of the total particle (dynamic+thermal) pressure over the magnetic pressure) was about ten times greater. This suggests that the jet was dominated by the particles.

\section{Spatial structure of the post-onset flow.}

The $B_{X}$ profile displayed in Figure 1 suggests that the lobe magnetic field value did not change significantly during the flow event. As confirmed by the plasma and spacecraft potential data (not shown), GEOTAIL completely exited from the plasma sheet into the south lobe at 07:06 UT for 3 minutes. The lobe magnetic field strength was similar to the one recorded before and after the event. This suggests that the tail structure persisted. Moreover, as the flow was roughly stationary between 06:15 and 08:30 UT, we may expect that the plasma sheet also maintained its structure along its normal direction $(Z)$ and roughly satisfied the 1-D pressure balance condition:

$$
\mathrm{P}_{\mathrm{S}}=\mathrm{P}_{\mathrm{m}}+\mathrm{P}_{\mathrm{th}}=\text { constant }
$$

where $P_{m}$ is the magnetic pressure, $P_{t h}$ is the thermal pressure and $P_{S}$ is the static pressure.

In order to test this assumption, panels (a) and (b) of Figure 3 provide comparison of the magnetic and the thermal pressures recorded from 06:15 to 08:30 UT. Panel (a) consists of a scatter plot of the magnetic pressure versus the thermal pressure, both taken at the highest (12 second) time resolution. They appear to be anti-correlated with a slope close to -1 . Panel (b) shows the magnetic, thermal and static pressures sampled over InT bins of $B_{\mathbf{X}}$. Both magnetic and thermal pressure profiles are quasisymmetrical relatively to the neutral sheet $\left(B_{X}=0\right)$. They are complementary in such a way that the static pressure took roughly constant values through the plasma sheet thickness. These observations are consistent with the 1-D pressure balance model of the plasma sheet, suggesting that it consists of an appropriate approximation to describe the large-scale features of the post-onset flow. Particularly, the general agreement of the observation with the 1-D pressure balance model supports the use of $B_{X}$ as an indicator of the distance to the neutral sheet and allows to estimate the instantaneous lobe Alfven velocity:

$$
V_{A, L}=\frac{B L}{\sqrt{\mu_{0} \cdot N L . m}}=\sqrt{\frac{2 . P S}{N L . m}}
$$
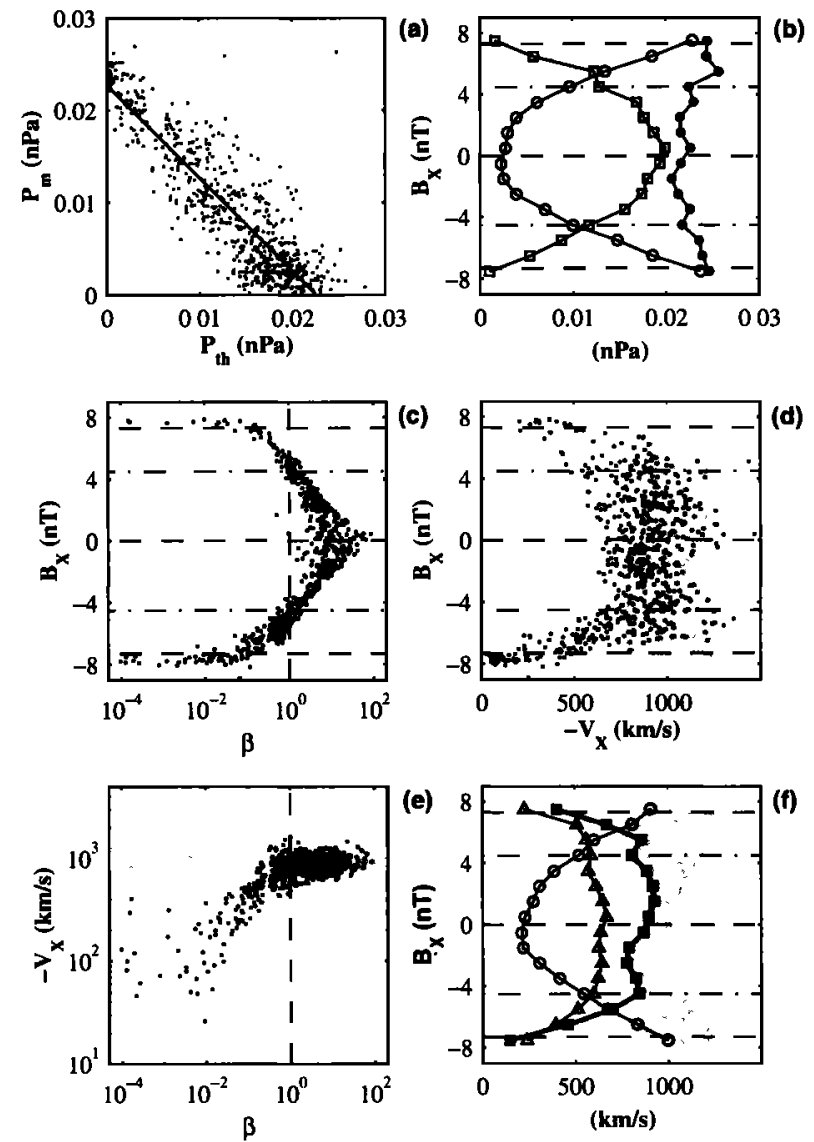

Figure 3. Data obtained onboard GEOTAIL (06:15-08:30 UT). Panel (a): Magnetic pressure versus thermal pressure; (b): Profiles of magnetic pressure (circles), thermal pressure (squares) and static pressure (dots), relatively to $B_{X}$. The dashed-dotted lines represent the transition layers at $\left|B_{X}\right| \sim 4.5$ nT (see text); (c): $\boldsymbol{\beta}$ plasma parameter profile; (d): Profile of the tailward component of the plasma velocity $\left(-V_{X}\right)$. The shadowed area represents the lobe Alfven velocity range; (e): $-V_{X}$ versus $\beta$; (f): Profile of $-V_{X}$ (squares), local Alfven speed (circles) and sound speed (triangles). 
Here, $m$ is the proton mass, $N_{L}$ represents the density in the lobe. $\mathrm{B}_{\mathrm{L}}$ is the lobe magnetic field strength. Taking values of $\mathrm{Ps}$ between 0.020 and $0.026 \mathrm{nPa}$ and values of $\mathrm{N}_{\mathrm{L}}$ between 0.02 and $0.04 \mathrm{~cm}^{-3}$, the lobe Alfven velocity is estimated to range from 780 to $1250 \mathrm{~km} / \mathrm{s}$.

Panel (c) displays the profile of the $\beta$ plasma parameter as a function of the plasma sheet thickness, using $\mathrm{B}_{\mathrm{X}}$ as indicator of the distance to the neutral sheet. It exhibits a quasi-symmetrical shape relative to the neutral sheet. In the following, we will distinguish the outer layers of the plasma sheet as the regions where $\beta$ is less than 1 and the central layer where $\beta$ is greater than 1 . The transition layers between central layer and outer layers occur at $\left|B_{x}\right| \sim 4.5 n T$ in both North and South plasma sheet and are represented by dashed-dotted horizontal lines.

Panel (d) shows the profile of the tailward plasma velocity ($V_{X}$ ). In the central layer, the profile is flat with values of the order of $900 \pm 200 \mathrm{~km} / \mathrm{s}$. These values are close to the lobe Alfven velocity represented by the shadowed area. In the outer layers, the plasma velocity gradually increases from the lobes to the transition layers. Thus, two flow regimes clearly appear, as shown in panel (e). For $\beta<1$, i.e. in the outer layers, the velocity increases with $\beta$ and for $\beta>1$, i.e. in the central layer, the velocity is roughly constant, of the same order of the lobe Alfven speed. In panel (f), $-V_{X}$ is compared to the speeds that are typical of the medium. The data have been averaged over $\ln T B_{X}$ intervals. It shows that the flow was supersonic and super-Alfvenic.

\section{Summary and conclusion.}

We report this event because (i) it was associated with an isolated substorm and (ii) the GEOTAIL satellite continuously stayed inside the plasma sheet during a long period following the substorm onset. Multi-instrument measurements show that the main signature of the substorm consisted of a fast tailward flow exhibiting the following features:

- the flow lasted more than two hours and a half. The scale of this tailward jet was very large, greater than $1000 R_{E}$, as inferred by multiplying its duration by the average plasma velocity;

- the tailward jet streamed between North and South lobes and conserved a plasma sheet like structure, which can be described at first order by a simple 1-D pressure balance. Accordingly, the $\mathrm{B}_{\mathrm{X}}$ component of the magnetic field could be used as an appropriate indicator of the distance to the neutral sheet and allowed to study the Z-structure of the jet;

- two clearly distinct regions appear, associated with distinct flow regimes. The transition between outer and inner layers precisely occurs through the layer where the $\beta$ plasma parameter is equal to 1 . In the central plasma sheet $(\beta>1)$, the plasma bulk velocity was uniform and quasi-stationary. It sustained a roughly constant value close to the lobe Alfven speed during more than two hours; - highly tailward oriented energetic ion fluxes were continuously detected during the event. Taking into account the values of the bulk flow velocity, they could consist of thermal populations in the central plasma sheet and non-thermal populations in the plasma sheet boundary layers. Further examination may answer this question;

- a close anti-correlation between $E_{Y}$ and $B_{Z}$ was observed up to the time resolution of the EFD instrument ( 3 seconds), suggesting that the flow can be described by the hydromagnetic approximation $(\mathbf{E}=-\mathbf{V x B})$.

Substorm associated fast tailward flow were observed with similar features by GEOTAIL at geocentric distances ranging between 90 and $210 \mathrm{R}_{\mathrm{E}}$. The stationarity of these plasma jets, their source, their spatial structure, the nature of the associated magnetic turbulence and the mechanism of particle acceleration are open issues which wll be investigated in future studies.

Acknowledgement. The authors are grateful to $T$. Hughes (CANOPUS/MARIA Network, Canadian Space Agency) for providing ground magnetogram data. The AL data were provided by the World Data Center C-2 for Geomagnetism. Stimulating comments from the PNST team are also gratefully acknowledged.

\section{References}

Hones, E.W., Jr. et al., Structure of the magnetotail at $220 \mathrm{Re}$ and its response to geomagnetic activity, Geophys. Res. Lett., 11, 5, 1984.

Jacquey, C., et al., Tailward energetic ion streams observed at $100 R_{E}$ by GEOTAIL-EPIC associated with geomagnetic activity intensification, Geophys. Res. Lett., 21, 3015, 1994.

Kokubun, S., et al., The GEOTAIL Magnetic Field Experiment, $J$. Geomag. Geoelectr., 46, 7, 1994.

Moldwin, M.B. and W.J. Hughes, Geomagnetic substorm association of plasmoids, , J. of Geophys. Res., 98, 81, 1993.

Mukai, T., et al., The Low Energy Particle (LEP) Experiment onboard the GEOTAIL satellite, J. Geomag. Geoelectr., 46, 669, 1994.

Mukai, T., et al., Dynamics and kinetic properties of plasmoids and flux ropes: GEOTAIL observations, New perspectives on the Earth's magnetotal, AGU Monogr. 105, 117, 1998.

Pedersen et al., Quasi-static electric field measurements with spherical double probes on the GEOS and ISEE satellites, Space Sci. Rev., 37, 269, 1984

Richardson, I.G., et al., Plasmoid-associated energetic ion bursts in the deep geomagnetic tail: properties of plasmoids and the postplasmoid plasma sheet, , J. of Geophys. Res., 92, 9997, 1987.

Tsuruda, K., et al., Electric field measurements on the GEOTAIL satellite, J. Geomag. Geoelectr., 46, 693, 1994.

Williams, D.J., Energetic ion beams at the edge of the plasma sheet: ISEE1 observations plus a simple explanatory model, J. of Geophys. Res., 86, 5507, 1981.

Williams, D.J., et al., GEOTAIL Energetic Particle and Ion Composition instrument, J. Geomag. Geoelectr., 46, 39, 1994.

S. Rouquette and C. Jacquey, CESR, 9 av. Col. Roche 31028 Toulouse, France. sebastien.rouquette@cesr.fr.

(Received April 28, 2000; revised July 28, 2000; accepted July 31,2000 ) 